\title{
Interactions Between Microglia and Newly Formed Hippocampal Neurons in Physiological and Seizure-Induced Inflammatory Environment
}

\author{
Deepti Chugh $^{\mathrm{a}, \mathrm{b}}$ and Christine T. Ekdahl ${ }^{\mathrm{a}, \mathrm{b}, *}$ \\ anflammation and Stem Cell Therapy Group, Division of Clinical Neurophysiology, Lund University, Lund, \\ Sweden \\ ${ }^{\mathrm{b}}$ Lund Epilepsy Center, Lund University, Lund, Sweden
}

\begin{abstract}
Adult hippocampal neurogenesis is modulated by physiological and pathological stimuli, including seizures and inflammation. Here, we describe stable interactions between microglia and newborn neurons using two-photon and confocal microscopy. On 3 weeks-old neurons, these interactions exhibit preferences for distal dendrites under physiological conditions. Conversely, after status epilepticus, ramified microglia, in particular, interact more with the proximal dendrites of new neurons. No such differences were found on 6 weeks-old neurons. Our study demonstrates regional and temporal specificity of the interactions between newborn neurons and microglia during a critical period for homeostasis and synaptic integration.
\end{abstract}

Keywords: Microglia, neurogenesis, hippocampus, seizures

\section{INTRODUCTION}

Adult neurogenic niche within the subgranular zone of the hippocampus is a highly specialized brain region capable of homeostatic plasticity. Newborn hippocampal neurons receive inhibitory synaptic inputs from hilar interneurons perisomatically in the granule cell layer (GCL) and on their proximal dendrites in the inner molecular layer (iML) of the dentate gyrus, while the excitatory inputs from the entorhinal cortex is found throughout the dendritic tree, including the distal dendrites in the middle/outer ML (mML/oML) [1]. Synaptic assembly starts at around 3 weeks, coinciding with a transition from

${ }^{*}$ Correspondence to: Christine T. Ekdahl, Associate Professor, MD, Inflammation and Stem Cell Therapy Group, Division of Clinical Neurophysiology, Lund Epilepsy Center, BMC A11, Sölvegatan 17, Lund University, SE-221 84 Lund, Sweden. Tel.: +46 46 2220550; Fax: +46 46 2220560; E-mail: Christine.Ekdahl_Clementson@med.lu.se. excitatory to inhibitory GABA signaling [1]. By 6 weeks, newborn neurons show several electrophysiological properties similar to mature granule cells [2]. Various pathological insults, including epileptic seizures, modulate adult neurogenesis. Seizures not only increase the production of newborn neurons but also influence their subsequent fate depending on the severity and the magnitude of the associated inflammatory response mediated by microglia [3-7].

Microglia, the innate immune cells of the brain, exhibit a dual role in adult neurogenesis. Under physiological conditions, microglia phagocytose apoptotic neuronal progenitor cells and promote neuronal differentiation via trophic factors [8, 9]. Conversely, following seizures, both detrimental and a supportive role for activated microglia have been described $[5,10]$. Microglia are also suggested to regulate synaptic integration of newborn neurons [11-13] by either direct physical interactions as demonstrated for microglia and mature neurons in the visual cor- 
tex $[14,15]$, or via intercellular signaling involving inflammatory mediators.

In the current study, we evaluated the physical interactions between microglia and newborn hippocampal neurons at early and late phases of synaptogenesis, using two-photon (2-p) and confocal microscopy. We also assessed if different microglial phenotypes exhibited region-specific preferences while interacting with newborn neurons.

\section{MATERIALS AND METHODS}

\section{Animals}

For 2-p experiments, 2-3 months old $\mathrm{CX} 3 \mathrm{CR} 1^{\mathrm{GFP} /+}$ heterozygous mice $(n=23$; originally bred by Dr. Steffen Jung, Rehvot, Israel, and kindly provided by Dr William Agace, Lund, Sweden) were used wherein the CX3CR1 locus encoding the chemokine receptor CX3CR1 was replaced with a reporter gene encoding enhanced green fluorescent protein (EGFP). For confocal microscopy, adult Sprague-Dawley rats $(n=19 ; 200-250 \mathrm{~g})$ were procured from Charles River (Germany). All experimental procedures were approved by the Malmö-Lund Ethical Committee in Sweden.

\section{Electrode implantation and induction of status epilepticus (SE) in rats}

Rats were implanted with bipolar electrodes unilaterally into hippocampus and 2 hours of SE was induced by electrical stimulation 7-days postimplantation as described previously [16].

\section{Retroviral labeling of new neurons}

For 2-p experiments, CX3CR1 $1^{\mathrm{GFP} /+}$ mice were injected under isofluorane anaesthesia with $1.5 \mu \mathrm{l}$ retroviral vector, expressing red fluorescent protein (RV-RFP) under the CAG promoter $\left(4.8^{*} 10^{8}\right.$ transducing units $/ \mathrm{ml})$, into the hippocampus $(2.0 \mathrm{~mm}$ caudal and $1.5 \mathrm{~mm}$ lateral to bregma, and $2.0 \mathrm{~mm}$ ventral to dura). For confocal microscopy, RV-GFP was injected in the hippocampus contralateral to the electrode 7 days following SE, as previously described [16].

\section{Immunohistochemistry}

For confocal microscopy, rats were perfused with ice-cold saline and $4 \%$ paraformaldehyde 3 or 6 weeks after retroviral injections. Brains were processed for immunohistochemistry as previously described [11]. The following primary and secondary antibodies were used: rabbit polyclonal anti-Iba1 (1:1000, Wako, Japan), chicken polyclonal anti-GFP (1:5000, Abcam, UK), mouse monoclonal anti-gephyrin (1:500, Synaptic Systems, Germany), Cy3-conjugated donkey anti-rabbit (1:200, Jackson Immunoresearch, UK), Cy3-conjugated goat anti-mouse (1:200, Jackson Immunoresearch), biotinylated goat anti-chicken (Vector laboratories), Cy5-conjugated goat anti-rabbit (1:200, Jackson Immunoresearch), Alexa-488 goat anti-chicken (1:200, Invitrogen, Sweden), and Alexa-488 streptavidin (1:200, Invitrogen).

\section{Confocal microscopy}

For GFP and Ibal immunostaining, images were acquired with $488 \mathrm{~nm}$ and $561 \mathrm{~nm}$ excitation lasers using a confocal microscope (Carl Zeiss, Germany) with 40x oil immersion objective in a z-stack at an interval of $1 \mu \mathrm{m}$. For GFP/gephyrin/Iba1 triple immunostaining, images were acquired with $63 \mathrm{x}$ oil immersion objective in a z-stack at an interval of $0.5 \mu \mathrm{m}$ using an additional $633 \mathrm{~nm}$ excitation laser for $\mathrm{Cy} 5$ detection.

\section{Brain slice preparation}

For 2-p experiments, CX3CR $1^{\mathrm{GFP} /+}$ mice at 3-4 weeks or 6-7 weeks after retroviral injections were decapitated. Brains were removed and coronal slices $(200 \mu \mathrm{m})$ were cut on a vibratome (Leica, Germany) in gassed $\left(95 \% \mathrm{O}_{2}\right.$ and $\left.5 \% \mathrm{CO}_{2}\right)$ ice-cold solution (in mM: 75 sucrose, $67 \mathrm{NaCl}, 26 \mathrm{NaHCO}_{3}, 25$ glucose, $2.5 \mathrm{KCl}, 1.25 \mathrm{NaH}_{2} \mathrm{PO}_{4}, 0.5 \mathrm{CaCl}_{2}, 7 \mathrm{MgCl}_{2}$ (pH:7.4, 295-300 mOsm)). Slices were immediately transferred to a chamber containing gassed artificial cerebrospinal fluid (in mM: $119 \mathrm{NaCl}, 26 \mathrm{NaHCO}_{3}$, 25 glucose, $2.5 \mathrm{KCl}, 1.25 \mathrm{NaH}_{2} \mathrm{PO}_{4}, 2.5 \mathrm{CaCl}_{2}$, $\left.1.3 \mathrm{MgSO}_{4}(\mathrm{pH}: 7.4,295-300 \mathrm{mOsm})\right)$ at $34^{\circ} \mathrm{C}$ and allowed to rest for $1 \mathrm{~h}$ before imaging.

\section{2-p microscopy}

Images of RFP-labeled newborn neurons and EGFP-labeled microglia in the hippocampus were acquired using 2-p microscopy (Carl Zeiss 710 NLO, Germany), with a MaiTai laser (Spectra Physics, Santa Clara, CA) tuned to $760 \mathrm{~nm}$ and a $63 \mathrm{x}$ waterimmersion objective in a z-stack at an interval of $1 \mu \mathrm{m}$. Continuous imaging was performed for 5-6 cycles for a total duration of $\sim 20 \mathrm{~min}$. 


\section{Image analysis}

Images were analyzed using three-dimensional (3D) image analysis software Volocity (Perkin Elmer). For 2-p images, interaction was defined when the region depicting the co-localization between RFP and EGFP signals appeared yellow in 3D-rendered images. This interaction depicts a close anatomical contact made by $\mathrm{EGFP}^{+}$microglial cells with the $\mathrm{RFP}^{+}$newborn neuron. A total of 50-70 RFP ${ }^{+}$cells were analyzed for 3-4 and 6-7 weeks time point, respectively.

For confocal images, interaction was similarly defined in terms of co-localization between Iba1 and GFP signals in granule cell layer (GCL), inner molecular layer (iML), and middle/outer ML (mML/oML). The first one-third of the ML was considered as iML and the last two-third was considered as mML/oML. For quantification, the number of microglia of each morphology that interact with the soma in the GCL, proximal dendrites in the iML, and the distal dendrites in the mML/oML were counted from a total of 5-10 images comprising a minimum of $10 \mathrm{GFP}^{+}$ newborn neurons per animal. Data is then expressed as relative percentage of microglia interacting in three regions of interest for both morphological subtypes (ramified and intermediate), respectively. Triple colocalization of GFP, gephyrin, and Iba1 was analyzed in proximal dendrites from a minimum of 10 newborn neurons per animal. The data for triple co-localization is expressed as the percentage of microglia-new neuron interactions that are closely associated with gephyrin.

\section{Statistical analysis}

Statistical analyses were performed with either unpaired students $t$-test or with two-way analysis of variance (ANOVA) when comparing two or multiple groups, respectively, using Graphpad Prism. Data are presented as mean \pm SEM, and differences were considered statistically significant at $p \leq 0.05$.

\section{RESULTS}

\section{Stable interactions between newborn} hippocampal neurons and microglia

We evaluated the dynamics of interactions between newborn neurons $\left(\mathrm{RFP}^{+}\right)$and microglia $\left(\mathrm{EGFP}^{+}\right)$ using 2-p microscopy under physiological conditions at two phases of synaptogenesis: 3-4 weeks and 6-7 weeks, representing the synaptic assembly and maturation phases, respectively (Fig. 1A and 1B). At both time points, interactions were mediated by ramified (Fig. 1C) and intermediate (Fig. 1D) microglia and very rarely by amoeboid/round microglia (Fig. 1E). Two kinds of interactions were evident: stable (Fig. 1F-J) and transient (Fig. 1K-O) during 10-20 min of scanning. In addition, there were some interactions that could not be classified as either stable or transient because the 2-p signal was bleached over time; those interactions are termed as "unclassified". About $60-70 \%$ of the interactions were stable at both time points (Fig. 1P) and these stable interactions were uniformly distributed in the GCL, iML, and mML/oML (Fig. 1Q).

\section{Microglia interact preferentially with the distal dendrites of 3-weeks old newborn neurons under physiological conditions}

We further evaluated the interactions by confocal microscopy of fixed brain sections from control rats. We investigated whether different microglial phenotypes interact differently with newborn neurons at 3 and 6 weeks. We observed that both ramified and intermediate microglia interacted significantly more with the distal dendrites in the mML/oML of 3weeks old neuron (Table 1). In contrast, at 6 weeks, the interactions by ramified cells were evenly distributed along the dendrites and soma; while those by intermediate cells were still significantly more in the mML/oML (Table 1).

\section{Ramified microglia interact preferentially with} the proximal dendrites of 3-weeks old newborn neurons following status epilepticus (SE)

Next, we compared the interactions in the seizureinduced pathological environment. Both ramified and intermediate cells interacted more with the dendrites compared to cell soma of 3 and 6 weeksold neurons (born 1 week after SE) (Table 1). Interestingly, the interactions were significantly increased $\left(\mathrm{F}_{(2,24)}=3.522, p=0.04\right)$ on the proximal dendrites in the iML of 3 weeks-old neurons following SE compared to non-stimulated controls (NSC) (Fig. 2A-C). The increase was represented by ramified $\left(\mathrm{F}_{(2,12)}=4.080, p=0.04\right)$ and not intermediate $\left(\mathrm{F}_{(2,21)}=0.3479, p=0.7\right)$ microglia (Table 1, Fig. 2D and 2E). No such differences were found on 6 weeks-old neurons $\left(\mathrm{F}_{(2,21)}=0.5308, p=0.59\right)$ (Fig. 2F). The total number of microglia interacting 

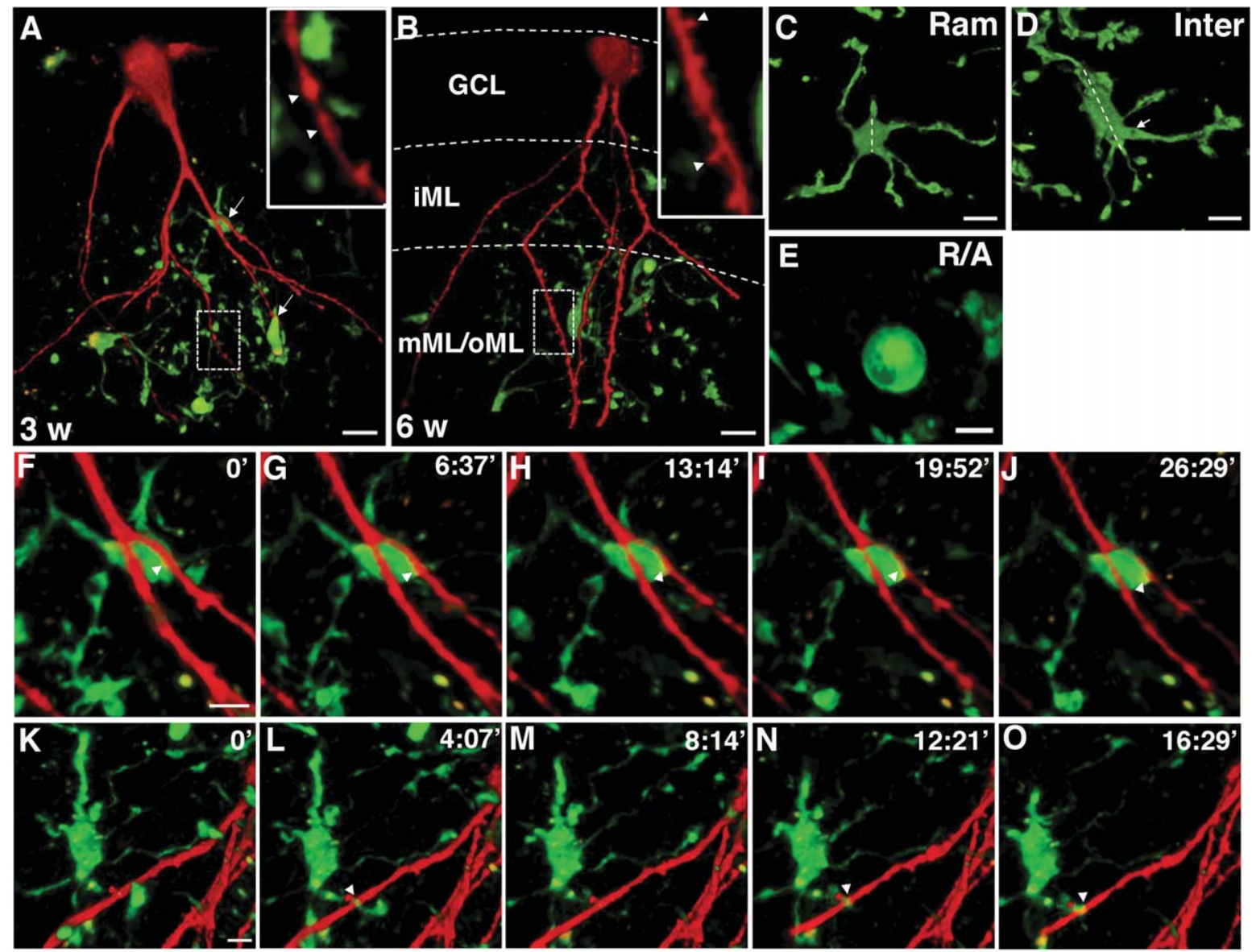

$\mathbf{P}$
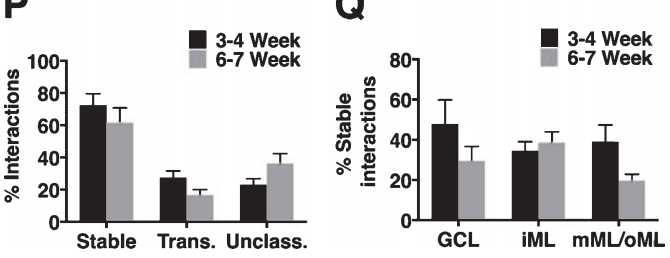

Fig. 1. A-B, Photomicrographs representing $\mathrm{EGFP}^{+}$microglia and $\mathrm{RFP}^{+}$newborn neurons at 3- (A) and 6- (B) weeks after RV-RFP injection in $\mathrm{CX} 3 \mathrm{CR} 1^{\mathrm{GFP} /+}$ mice (arrows indicate interactions in A, arrow heads indicate dendritic beading at 3 weeks in A and spines at 6 weeks in B). C-E, Images showing different morphologies of microglia: ramified (Ram; C), intermediate (Inter; D) and round/amoeboid (R/A; E). Dotted lines in C and D outline the largest diameter of the cell soma. Note the elongated shape of cell soma and the thicker processes (marked by an arrow) of an intermediate microglia in D. F-O, 2-p images showing the dynamics of interactions between newborn neuron and microglia (arrowheads indicate stable interaction in F-J and a transient interaction in K-O). P, Quantification of relative percentage of stable, transient, and unclassified interactions in 3 and 6 weeks-old neurons. Q, Quantification of relative percentage of stable interactions in 3 and 6 weeks-old neurons in GCL, iML, and mML/oML. Scale bars: $13.55 \mu \mathrm{m}$ (A,B), $9.03 \mu \mathrm{m}(\mathrm{C}, \mathrm{D}), 6.77 \mu \mathrm{m}$ (E), and $6.77 \mu \mathrm{m}$ (in F for F-J and in K for K-O). GCL: granule cell layer, iML: inner molecular layer, mML/oML: middle/outer molecular layer, Trans: transient, Unclass: unclassified. $n=10$ mice for 3-4 weeks group and $n=7$ mice for 6-7 weeks group.

per soma/dendrite of the newborn neuron in the iML was, however, unaltered (Table 2). Instead, we found a small trend towards an increase in the number of proximal dendrites in the iML on 3 weeks-old neurons following SE (NSC: $4.7 \pm 0.68$ vs SE $7.6 \pm 1.42$, $p=0.1)$.
Since the proximal dendrites in the iML primarily receive inhibitory synaptic inputs, we also investigated if microglial interactions were specifically associated with inhibitory synapses on the newborn neurons. A triple co-localization analysis of GFP, gephyrin (an inhibitory post-synaptic scaf- 
Table 1

Quantification of relative percentage of microglia interacting with the 3- and 6-weeks old newborn neurons in the granule cell layer (GCL), inner molecular layer (iML), and middle/outer molecular layer (mML/oML) in non-stimulated controls (NSC) and after status epilepticus (SE). Data are presented as mean \pm SEM, $n=3-5$ animals for each group (10-15 $\mathrm{GFP}^{+}$newborn neurons per animal). ${ }^{*}-$ significant difference between NSC and SE groups in relative percentage of ramified microglia interacting with the proximal dendrites of 3-weeks old neurons in iML by 2-way ANOVA $\left(\mathrm{F}_{(2,12)}=4.080, p=0.04\right)$

\begin{tabular}{|c|c|c|c|c|c|c|}
\hline \multirow[t]{3}{*}{ Groups } & \multicolumn{6}{|c|}{$\%$ microglia interacting in dentate gyrus } \\
\hline & \multicolumn{3}{|c|}{ Ramified } & \multicolumn{3}{|c|}{ Intermediate } \\
\hline & GCL & iML & $\mathrm{mML} / \mathrm{oML}$ & GCL & iML & mML/oML \\
\hline 3 w-old neurons (NSC) & $14.4 \pm 5.3$ & $22.3 \pm 1.6$ & $63.2 \pm 6.7$ & $10.7 \pm 4.2$ & $32.3 \pm 5.0$ & $57.0 \pm 7.7$ \\
\hline 3 w-old neurons (SE)* & $11.4 \pm 9.0$ & $50.4 \pm 16.5$ & $38.2 \pm 9.8$ & $5.8 \pm 2.6$ & $32.0 \pm 6.2$ & $62.2 \pm 8.3$ \\
\hline $6 \mathrm{w}$-old neurons (NSC) & $25.8 \pm 19.7$ & $47.1 \pm 16.9$ & $27.1 \pm 11.5$ & $2.1 \pm 2.1$ & $34.3 \pm 12.4$ & $63.7 \pm 12.8$ \\
\hline $6 \mathrm{w}$-old neurons (SE) & $3.3 \pm 3.3$ & $48.9 \pm 25.8$ & $47.8 \pm 24.8$ & $11.3 \pm 5.9$ & $56.0 \pm 22.0$ & $32.7 \pm 16.4$ \\
\hline
\end{tabular}

folding protein) and Iba1 (Fig. $2 \mathrm{G}$ and $2 \mathrm{H}$ ) revealed that almost $40 \%$ of the anatomical contacts made by microglia were associated with gephyrin clusters present on the proximal dendrites of the newborn neurons in both NSC and SE animals (NSC: $36.01 \pm 6.50$ $\%$ vs SE $38.21 \pm 6.65 \%$ ).

\section{DISCUSSION}

Here, we demonstrate direct region-specific interactions between microglia and newborn neurons at the time when new neurons integrate synaptically into the adult hippocampal circuitry. We also find that these interactions change following seizures.

The majority of interactions were stable at two distinct phases of synaptogenesis ( 3 and 6 weeks). This is in contrast to previously described transient interactions between microglia and mature neurons lasting for an average duration of $5 \mathrm{~min}$ under physiological conditions [15], and may reflect differential responses depending on neuronal age $[7,16]$. However, due to the long scanning sequences used in the present study, more rapid and dynamic changes cannot be ruled out.

The observed temporal change in the preferential localization of interactions by ramified microglia, from distal dendrites to a more uniform distribution along the soma and dendrites, correlates well with the temporal course of synaptic development where dendritic inputs precede perisomatic inputs onto newborn neuron under physiological conditions [17]. Interestingly, this sub-regional localization changed significantly following SE in 3-weeks old neurons. Ramified microglia interacted more with the proximal dendrites, which may reflect increased involvement of microglia in synaptic reorganization and pruning in the iML. Indeed, both recurrent basal dendrites and mossy fiber sprouting are fre- quently observed in the iML following SE [4] and we did observe a small trend for increased numbers of dendrites in the iML. In addition, since the iML receives primarily inhibitory inputs, which also change following seizures [16], it is intriguing to speculate that the microglial interactions may be directed towards inhibitory synapses. Indeed, microglia interact directly with axon-initial segments of mature neurons, a region that receives mainly inhibitory synapses [18]. Our data with $\sim 40 \%$ of the microglia-new neuron contacts being associated with gephyrin clusters further supports a significant amount of interaction with inhibitory synapses. However, the close anatomical association of microglia-new neuron contacts with gephyrin did not change following seizures. This was partly expected since the over-all number of gephyrin clusters is relatively small at this early developmental stage compared to the expression on 6-7 weeks-old neurons [16].

Conversely, the intermediate microglia continued with their regional preferences for distal dendrites in controls and SE animals at both time points, which may suggest diverse functions of these interactions, compared to those of ramified microglia. Absence of any changes in interactions on more mature 6-weeks old neurons following SE is consistent with our recent study where an LPS-induced inflammatory environment modulated synaptic properties of 4-weeks but not 7-weeks old newborn neurons [11], and support an important role for physical interactions between microglia and newborn neurons particularly during early synaptic development.

Our findings provide evidence for region- and time-dependent interactions between microglia and newborn neurons during synaptogenesis. They contribute towards further understanding of an important 

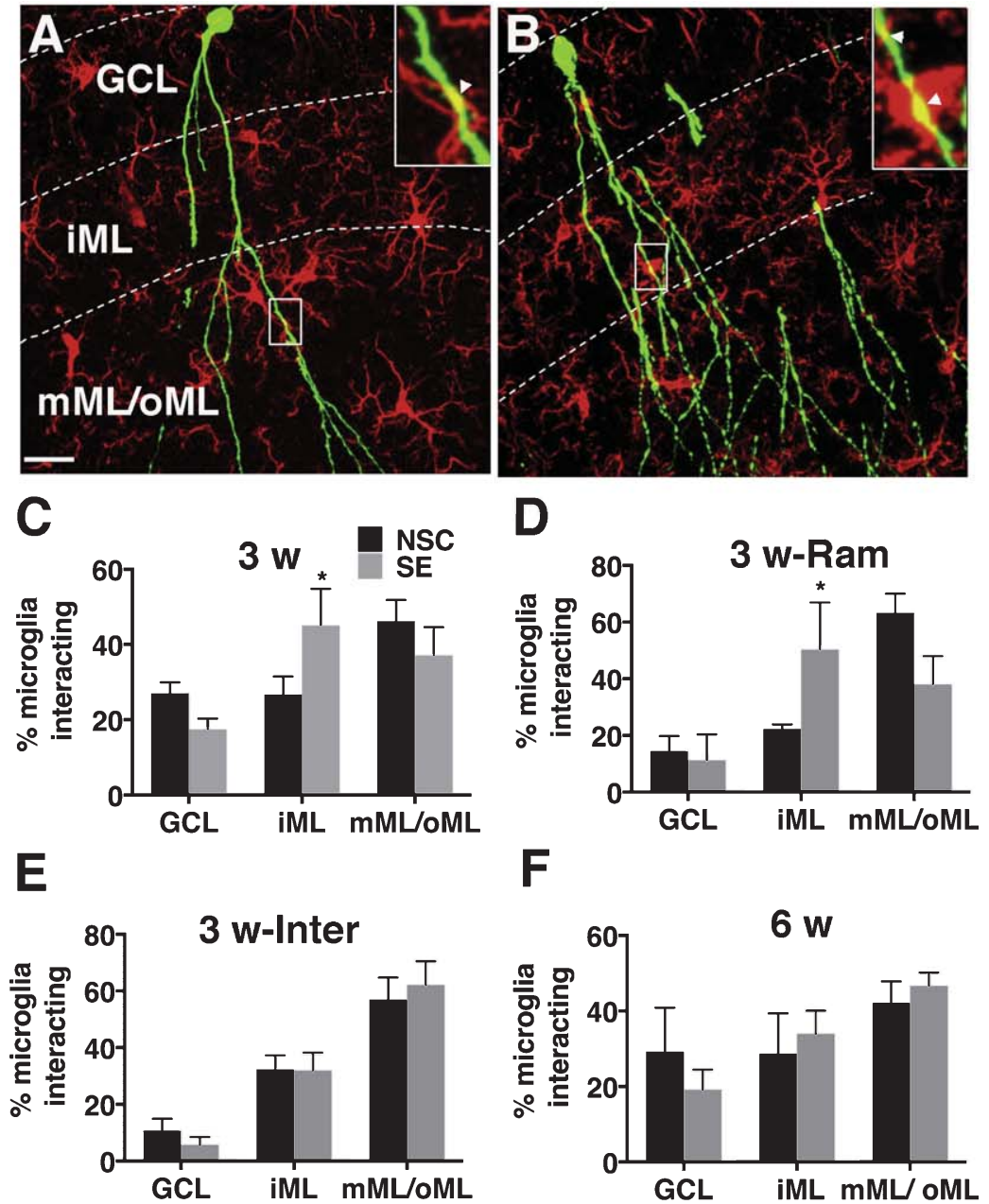

$\mathbf{F}$
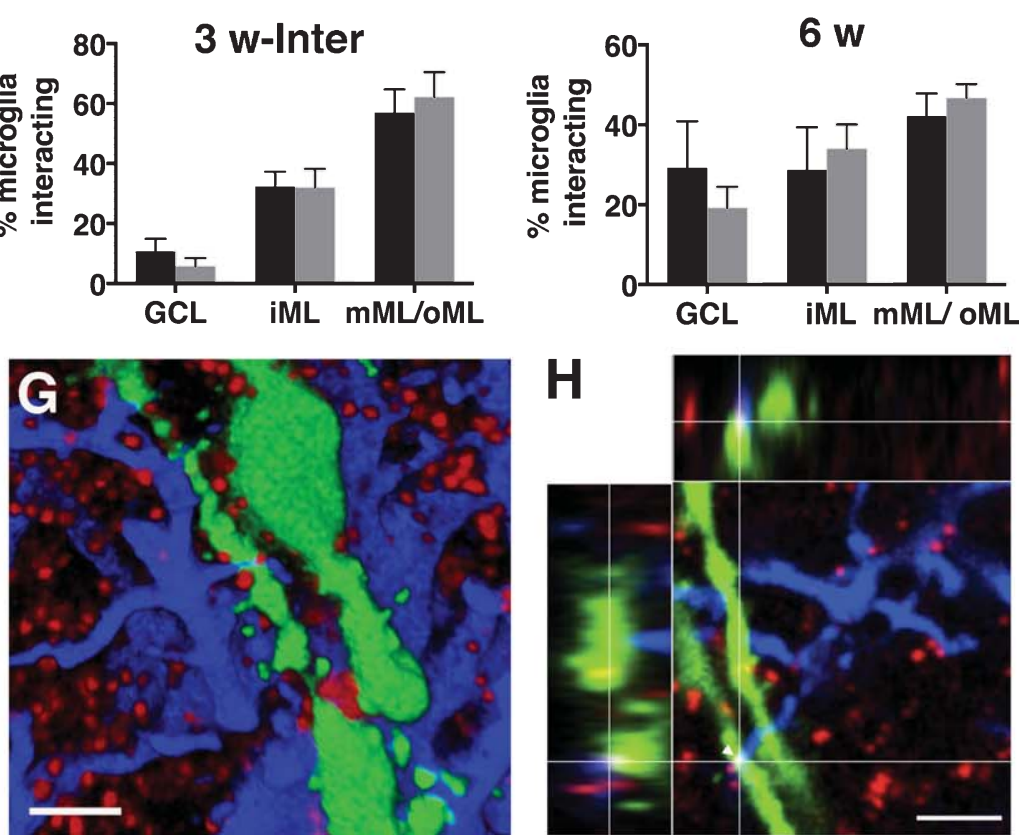

Fig. 2. A-B, Confocal photomicrographs representing Iba1 ${ }^{+}$microglia (red) and 3-weeks old $\mathrm{GFP}^{+}$newborn neurons (green) in NSC (A) and SE (B) rat. Insets in A and B depict zoomed images of microglia-new neuron interaction as marked by arrowheads. C-F, Quantification of relative percentage of microglia interacting in GCL, iML, and mML/oML with 3 weeks-old neurons $(\mathrm{C}$, all microglia; $\mathrm{D}$, ramified and $\mathrm{E}$, intermediate microglia) and 6 weeks-old neurons (F, all microglia) in NSC and SE animals. G-H, Representative confocal photomicrograph showing a close association of microglia (blue) with gephyrin clusters (red) present on the proximal dendrite of 3-weeks old newborn neuron (green) in a 3-D rendered image $(\mathrm{G})$ and in a 2-D image showing triple co-localization confirmed with orthogonal projection (H). Scale bars- $24 \mu \mathrm{m}$ (in A for A-B), $3.6 \mu \mathrm{m}(\mathrm{G})$, and $3.3 \mu \mathrm{m}(\mathrm{H})$. Data are presented as mean \pm SEM, $n=3-5$ animals for each group (10-15 GFP ${ }^{+}$ newborn neurons per animal), ${ }^{*}$ - significant difference between NSC and SE groups in relative percentage of microglia interacting with newborn neurons by 2-way ANOVA, $p \leq 0.05$. 
Table 2

Quantification of absolute number of microglia interacting per soma or per dendrite of 3- and 6-weeks old newborn neurons in GCL, iML, and mML/oML, respectively in NSC and after SE. Data are presented as mean $\pm \mathrm{SEM}, n=3-5$ animals for each group (10-15 $\mathrm{GFP}^{+}$newborn neurons per animal)

\begin{tabular}{lccc}
\hline Groups & GCL & iML & mML/oML \\
\hline 3 w-old neurons (NSC) & $0.9 \pm 0.3$ & $0.5 \pm 0.2$ & $0.6 \pm 0.1$ \\
3 w-old neurons (SE) & $0.9 \pm 0.4$ & $0.4 \pm 0.1$ & $0.5 \pm 0.2$ \\
6 w-old neurons (NSC) & $0.7 \pm 0.2$ & $0.5 \pm 0.2$ & $0.5 \pm 0.1$ \\
6 w-old neurons (SE) & $0.5 \pm 0.1$ & $0.3 \pm 0.08$ & $0.3 \pm 0.1$ \\
\hline
\end{tabular}

yet less explored possible role of microglia in regulating synaptic integration of newborn neurons. Additionally, it has important implications for improving cell-replacement therapies.

\section{ACKNOWLEDGMENTS}

We thank Dr. Johanna Jackson for performing parts of the surgeries. This work was supported by the Swedish Research Council, ALF Grant for funding for medical training and research, Zoega's, Tore Nilson's, and Åhlens's foundations. The research leading to these results has also received funding from the European Union's Seventh Framework Program (FP7/2007-2013) under grant agreement $n^{\circ} 602102$ (EPITARGET).

\section{CONFLICTS OF INTEREST}

The authors have no conflicts of interest to report.

\section{REFERENCES}

[1] Toni N, Sultan S. Synapse formation on adult-born hippocampal neurons. Eur J Neurosci. 2011;33(6):1062-8.

[2] van Praag H, Schinder AF, Christie BR, Toni N, Palmer TD, Gage FH. Functional neurogenesis in the adult hippocampus. Nature. 2002;415(6875):1030-4.

[3] Bengzon J, Kokaia Z, Elmer E, Nanobashvili A, Kokaia M, Lindvall $\mathrm{O}$. Apoptosis and proliferation of dentate gyrus neurons after single and intermittent limbic seizures. Proc Natl Acad Sci U S A. 1997;94(19):10432-7.

[4] Parent JM, Yu TW, Leibowitz RT, Geschwind DH, Sloviter RS, Lowenstein DH. Dentate granule cell neuro- genesis is increased by seizures and contributes to aberrant network reorganization in the adult rat hippocampus. J Neurosci. 1997;17(10):3727-738.

[5] Ekdahl CT, Claasen JH, Bonde S, Kokaia Z, Lindvall O. Inflammation is detrimental for neurogenesis in adult brain. Proc Natl Acad Sci U S A. 2003;100(23):13632-37.

[6] Mohapel P, Ekdahl CT, Lindvall O. Status epilepticus severity influences the long-term outcome of neurogenesis in the adult dentate gyrus. Neurobiol Dis. 2004;15(2):196-205.

[7] Jakubs K, Nanobashvili A, Bonde S, Ekdahl CT, Kokaia Z, Kokaia M, Lindvall O. Environment matters: Synaptic properties of neurons born in the epileptic adult brain develop to reduce excitability. Neuron. 2006;52(6):1047-59.

[8] Sierra A, Encinas JM, Deudero JJP, Chancey JH, Enikolopov G, Overstreet-Wadiche LS, Tsirka SE, Maletic-Savatic M. Microglia Shape Adult Hippocampal Neurogenesis through Apoptosis-Coupled Phagocytosis. Cell Stem Cell. 2010;7(4):483-95.

[9] Walton NM, Sutter BM, Laywell ED, Levkoff LH, Kearns SM, Marshall GP, 2nd, Scheffler B, Steindler DA. Microglia instruct subventricular zone neurogenesis. Glia. 2006;54(8):815-25.

[10] Bonde S, Ekdahl CT, Lindvall O. Long-term neuronal replacement in adult rat hippocampus after status epilepticus despite chronic inflammation. Eur $\mathrm{J}$ Neurosci. 2006;23(4):965-74.

[11] Chugh D, Nilsson P, Afjei SA, Bakochi A, Ekdahl CT. Brain inflammation induces post-synaptic changes during early synapse formation in adult-born hippocampal neurons. Exp Neurol. 2013;250:176-88.

[12] Ekdahl CT. Microglial activation - tuning and pruning adult neurogenesis. Front Pharmacol. 2012;3:41

[13] Jakubs K, Bonde S, Iosif RE, Ekdahl CT, Kokaia Z, Kokaia $\mathrm{M}$, Lindvall $\mathrm{O}$. Inflammation regulates functional integration of neurons born in adult brain. J Neurosci. 2008;28(47): 12477-88

[14] Tremblay ME, Lowery RL, Majewska AK. Microglial Interactions with Synapses Are Modulated by Visual Experience. Plos Biology. 2010;8(11):e1000527.

[15] Wake H, Moorhouse AJ, Jinno S, Kohsaka S, Nabekura J. Resting microglia directly monitor the functional state of synapses in vivo and determine the fate of ischemic terminals. J Neurosci. 2009;29(13):3974-80.

[16] Jackson J, Chugh D, Nilsson P, Wood J, Carlstrom K, Lindvall O, Ekdahl CT. Altered synaptic properties during integration of adult-born hippocampal neurons following a seizure insult. PLoS One. 2012;7(4):e35557.

[17] Esposito MS, Piatti VC, Laplagne DA, Morgenstern NA, Ferrari CC, Pitossi FJ, Schinder AF. Neuronal differentiation in the adult hippocampus recapitulates embryonic development. J Neurosci. 2005;25(44):10074-86.

[18] Baalman K, Marin MA, Ho TS, Godoy M, Cherian L, Robertson C, Rasband MN. Axon initial segment-associated microglia. J Neurosci. 2015;35(5):2283-92. 